\title{
Gamma beam industrial applications at ELI-NP
}

\author{
Gabriel Suliman*, Violeta Iancu ${ }^{\dagger}$ and Calin A. Ur ${ }^{\ddagger}$ \\ Extreme Light Infrastructure - Nuclear Physics/ Horia Hulubei National Institute for R\&D in Physics and \\ Nuclear Engineering, 30 Reactorului Street, P.O.B. MG-6, Bucharest-Magurele, Judet Ilfov, RO-077125, \\ Romania \\ "gabriel.suliman@eli-np.ro, ’violeta.iancu@eli-np.ro, ${ }^{\dagger}$ calin.ur@eli-np.ro
}

Mihai Iovea

ACCENT PRO 2000, s.r.l., Nerva Traian 1, K6, Apt 26, Bucharest, S3, RO-031041, Romania
office@accent.ro

Izuru Daito $^{\S}$ and Hideaki Ohgaki ${ }^{\Uparrow}$

The Institute for Advanced Energy Kyoto University, Uji, Kyoto 6110011, Japan

§daito.izuru@gmail.com, 'ohgaki.hideaki.2w@kyoto-u.ac.jp

Published 1 September 2016

\begin{abstract}
The Nuclear Physics oriented pillar of the pan-European Extreme Light Infrastructure (ELI-NP) will host an ultra-bright, energy tunable, and quasi-monochromatic gamma-ray beam system in the range of $0.2-19.5 \mathrm{MeV}$ produced by laser Compton backscattering. This gamma beam satisfies the criteria for large-size product investigations with added capabilities like isotope detection through the use of nuclear resonance fluorescence (NRF) and is ideal for non-destructive testing applications. Two major applications of gamma beams are being envisaged at ELI-NP: industrial applications based on NRF and industrial radiography and tomography. Both applications exploit the unique characteristics of the gamma beam to deliver new opportunities for the industry. Here, we present the experimental setups proposed at ELI-NP and discuss their performance based on analytical calculations and GEANT4 numerical simulations. One of the main advantages of using the gamma beam at ELI-NP for applications based on NRF is the availability of an advanced detector array, which can enhance the advantages already provided by the high quality of the gamma beam.
\end{abstract}

Keywords: Gamma beam system; industrial tomography; nuclear resonance fluorescence.

\section{Introduction}

The nuclear physics oriented pillar of the pan-European Extreme Light Infrastructure (ELI-NP) will comprise two major research instruments: a high-power laser system and a very brilliant gamma beam system. ${ }^{1-4}$ The gamma beam system (GBS) was designed to deliver a very intense and brilliant gamma beam produced by the inverse Compton

This is an Open Access article published by World Scientific Publishing Company. It is distributed under the terms of the Creative Commons Attribution 3.0 (CC-BY) License. Further distribution of this work is permitted, provided the original work is properly cited. 
scattering of laser pulses on relativistic electrons (Laser Compton Backscattering - LCS). The main features of the gamma beam available at ELI-NP will be: high spectral density (about $10^{4}$ photons/s/eV), narrow bandwidth $(<0.5 \%)$ of tunable energy, and high degree of polarization $(>95 \%)^{5}$

The research program of ELI-NP includes both fundamental research and applied science. Industrial tomography, nuclear waste management, and medical applications can take advantage of the unique features of the gamma beam system to deliver new opportunities for industry and medical research. By using this gamma source, it will also be possible to detect and/or measure nuclides in an object non-destructively, which is a key technology for nuclear industrial applications such as management of radioactive wastes, nuclear material accounting, and safeguards.

With the new developments in the production of high-energy gamma beams by LCS, the interest in using computed tomography (CT) for industrial purposes has recently increased. ${ }^{6,7}$ Today, there are several laboratories in the world that can produce highenergy gamma beams by LCS and only few of these that can deliver high intensities as well. ${ }^{6,8}$ The future gamma system at ELI-NP, which will deliver both high-intensity and high-energy gamma beams, will be a perfect solution for industrial applications in tomography. ${ }^{3}$ The collimated beam intensity is a few orders of magnitude higher than at any other gamma-ray source available worldwide, substantially increasing the penetration length and the maximum size of investigated objects. In addition, the small beam width allows high resolution imaging for in-depth large-object investigations useful in bonding in aeronautics, welding and machining accuracy in the automotive industry, and in construction. ${ }^{3,6}$ A tunable-energy gamma beam is very useful for adapting the energy range with the scanned object composition to correctly reveal the distribution of differently attenuating materials like plastic, ceramic, and/or metal parts. Based on this feature, the dual/multi-energy technique could be also applied for scanning an object at different energies and obtaining information about its component materials.

In this paper, we describe the possibility to use the gamma beam from ELI-NP for industrial applications. Non-destructive assay based on nuclear resonance fluorescence (NRF) of nuclear materials will be performed using the high-intensity gamma beam and the high efficiency detector array available in ELI-NP. The simulation study for industrial applications in radiography and tomography will also be presented.

\section{Nuclear Resonance Fluorescence Based Applications}

Nuclear resonance fluorescence (NRF) is an attractive non-destructive analysis (NDA) method because it provides signatures for a wide variety of materials that can be used to characterize the irradiated samples. Its use for detecting special nuclear materials (SNM) within cargo containers was proposed by Bertozzi et al. ${ }^{9}$ and subsequently developed by several organizations, which have explored various research issues related to the application of NRF. ${ }^{10,11}$

The applicability of the NRF method today is quite general and broad. ${ }^{9-18}$ Apart from SNM and nuclear safeguards applications, ${ }^{12,13}$ Beck et al. ${ }^{14}$ used NRF to determine the 
concentration of ${ }^{13} \mathrm{C}$ in a diamond sample, Lakshmanan et al. ${ }^{15}$ proposed to use NRF imaging for visualizing breast cancer lesions, and recently a $2 \mathrm{D}$ image of the isotope distribution of ${ }^{208} \mathrm{~Pb}$ was demonstrated by Toyokawa et al. ${ }^{7}$ In the future, a nondestructive measurement of heavy element contaminants in food can also be envisioned.

So far, the most commonly used detection schemes for NRF-based investigations are the scattering and the transmission methods. In both cases, interrogating photons are used to induce resonant absorption, and the de-excitation photons are detected either in a backscattering geometry or in a self-absorption (transmission) geometry. ${ }^{16}$ The applications described here take into consideration both measuring schemes.

\subsection{ELI-NP array of detectors (ELIADE)}

One of the main advantages of using the gamma beam at ELI-NP for applications based on NRF is the availability of an advanced detector array, which can enhance by an order of magnitude the advantages stemming from the high quality of the gamma beam.

The proposed ELI-NP Array of Detectors (ELIADE) ${ }^{19}$ is composed of eight segmented clover detectors placed on two rings, one at 98 degrees and another at 135 degrees with respect to the photon beam direction. Similar clover detectors have been operated at TIGRESS since 2009. ${ }^{20,21}$ A schematic representation of the ELIADE setup in the configuration that will be used for NRF experiments is presented in Fig. 1 as it is viewed from GEANT4 $4^{22,23}$ simulations. This configuration is optimized for very accurate measurements, even at the cost of longer experiments, i.e. for thin targets. Measurements rely on the segmentation of the detectors to deal with the background (Compton-scattered and $511 \mathrm{keV}$ photons from the target). ${ }^{19,24}$ For applications, we are interested in obtaining the highest signal/noise ratio and an experimental time as short as possible. Therefore, for the NRF transmission experiments, we use much thicker targets at the center of the ELIADE array (used as the notch detector). For NRF scattering experiments, the object of interest will be placed at the center of the ELIADE array, which limits the size of the object to be investigated.

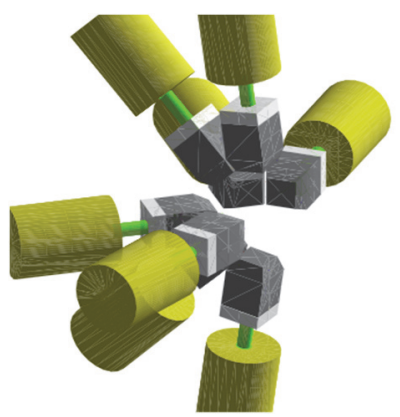

Fig. 1. 3D rendering of the ELIADE setup.

The gamma beam of ELI-NP has some very specific characteristics. ${ }^{5}$ As a result of the electron beam structure and the laser recirculation time, the photons are emitted at the 
interaction point with very high intensity and in very short duration pulses. At design parameters, $10^{8}$ photons reach the target every second, grouped in 100 macrobunches of $10^{6}$ photons each. ${ }^{5}$ Even in the extraordinary bandwidth conditions of ELI-NP $(0.5 \%$ of RMS energy), the number of photons within a typical nuclear resonance width $(1 \mathrm{eV})$ is only of the order of $10^{4}$ photons $/ \mathrm{s} / \mathrm{eV}$. In these conditions, it is crucial to estimate the number of photons being scattered from the target towards the detectors.

The two main processes that generate scattered photons in the target that travel toward the detectors are the Compton effect and pair generation in the nuclear field. We estimated the background coming from these effects with a $2 \mathrm{~cm}$ thick target of lead, which is one of the worst-case scenarios due to its high density and high Z. Calculations show that by using adequately thick $\mathrm{Pb}$ shields in front of the detectors, the number of these photons reaching the detector can be reduced by a few orders of magnitude to a level where a segmented detector can be employed without significant pile-up within each segment. ${ }^{19,24}$ Note that the background radiation is of low energy, so the frontal segments of the crystal will stop it with high probability.

The impact of the shield $(2 \mathrm{~cm} \mathrm{~Pb})$ on the absolute photo-peak efficiency and on the peak-to-total ratio is assessed using GEANT4 simulations of the clover detector with monoenergetic point sources and shown in Fig. 2. The passive lead filter has a higher impact on the low energy background photons than on the high-energy NRF photons, provided that the NRF signatures of the nuclei of interest are already known.
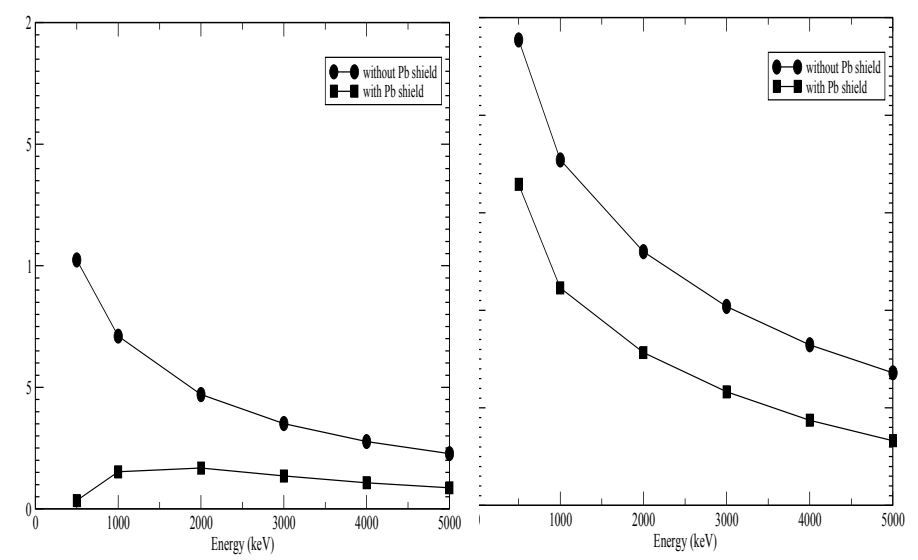

Fig. 2. The absolute efficiency and the peak-to-total ratio of a single clover detector.

\subsection{Analytical calculations}

An analytical model, described in Ref. 18, was used to compare the expected NRF rates for both transmission and scattering experiments with those experimentally measured at HI $\gamma \mathrm{S} .{ }^{11}$ The case study is the detection of SNM that are hidden in high-density materials using the same experimental setup as in Ref. 11. In the numerical examples, we used 
depleted uranium (DU) as a surrogate for actinides of interest and $\mathrm{W}$ or $\mathrm{Pb}$ as a surrogate for the matrix in which these actinides reside.

Hagmann et al. ${ }^{11}$ show that the total number of NRF events is 110 after 25.5 hours of measurement and concludes that a six-hour measurement would be required for a sixsigma level detection of sensitive material in the object when using the 100 photons/s/eV flux at $\mathrm{HI} \gamma \mathrm{S}$.

Table 1 shows the expected NRF rates estimated for the experimental conditions at ELI-NP and lists a few important parameters. Our estimates indicate that in the most unfavorable case, the same statistics as in the experiment at $\mathrm{HI} \gamma \mathrm{S}$ can be reached in less than two minutes. The difference of three orders of magnitude can be roughly attributed to the two orders of magnitude difference in the gamma-beam intensity and one order of magnitude from the detector-array efficiency. Note that the setup at ELI-NP includes a $2 \mathrm{~cm}$ thick absorber placed between the detectors and the notch target to attenuate the background radiation, as opposed to the $0.5 \mathrm{~cm}$ absorber used at $\mathrm{HI} \gamma \mathrm{S}$.

Table 1. Expected NRF rates for experimental conditions at ELI-NP. The parameters listed in the table are: the energy of the photons $(E)$, the flux of photons () , the mass $(m)$ and volume $(V)$ of the notch target, the thickness $(t)$ of the notch target, the probability that the NRF rays penetrate the lead filter $\left(P_{f}\right)$, the detector efficiency $(\varepsilon)$, and the number of detectors $\left(\mathrm{N}_{\mathrm{det}}\right)$. The estimations are made using the integrated cross section of $82 \mathrm{eV} \mathrm{b}$ for the $2.1 \mathrm{MeV}$ resonance of ${ }^{238} \mathrm{U}$.

\begin{tabular}{llllll}
\hline & No object & $1.3 \mathrm{~cm} \mathrm{~W}$ & $1.3 \mathrm{~cm} \mathrm{DU}$ & $\begin{array}{l}1.3 \mathrm{~cm} \mathrm{~W}+ \\
1.3 \mathrm{~cm} \mathrm{DU}\end{array}$ & Notes \\
\hline $\mathrm{E}(\mathrm{MeV})$ & 2.1 & 2.1 & 2.1 & 2.1 & \\
$\mathrm{~m}(\mathrm{~g})\left(\mathrm{UO}_{2}\right)$ & 1.88 & 1.88 & 1.88 & 1.88 & notch target \\
$\mathrm{V}\left(\mathrm{cm}^{3}\right)$ & 0.10 & 0.10 & 0.10 & 0.10 & notch target \\
Beam spot diameter $(\mathrm{cm})$ & 0.5 & 0.5 & 0.5 & 0.5 & notch target diameter \\
$\mathrm{t}(\mathrm{cm})$ & 0.50 & 0.50 & 0.50 & 0.50 & notch target \\
& & & & & thickness \\
\multicolumn{1}{c}{$\left(\right.$ photons/s/ $\left.\Gamma_{\mathrm{D}}\right)$} & 50000 & 50000 & 50000 & 50000 & all photons \\
$\quad \mathrm{i}\left(\right.$ photons $\left./ \mathrm{s} / \Gamma_{\mathrm{D}}\right)$ & 50000 & 16755 & 388 & 130 & resonant photons \\
$\mathrm{P}_{\mathrm{f}}$ & 0.36 & 0.36 & 0.36 & 0.36 & 2-cm lead filter \\
$\varepsilon(\%)$ & 0.3 & 0.3 & 0.3 & 0.3 & \\
$\mathrm{~N}_{\text {det }}$ & 8 & 8 & 8 & 8 & $\mathbf{1}$ \\
$\mathbf{R}_{\mathrm{NRF}}(\mathbf{c o u n t s} / \mathbf{s})$ & $\mathbf{3 1 1}$ & $\mathbf{1 0 4}$ & $\mathbf{2}$ & & \\
\hline
\end{tabular}

${ }^{*}$ The estimated number of photons coming from the source within the $1.78 \mathrm{eV}$ Debye width of the resonance $\left({ }^{238} \mathrm{U}\right)$ for a spectral density of $2.8 \times 10^{4}$ photons $/ \mathrm{s} / \mathrm{eV}$.

\section{Industrial Radiography and Tomography}

A radiography and tomography setup at ELI-NP will allow investigation of industrialsized objects with high resolution and high-contrast sensitivity. This setup will specialize in non-destructive experiments and analysis by performing 2D transmission images and $3 \mathrm{D}$ reconstructed tomography of the scanned objects, revealing the internal fine structure 
and composition. Moreover, this setup may also be employed in performing isotopespecific imaging/mapping through the use of the nuclear resonance fluorescence (NRF) technique, as has been recently demonstrated by Daito et al. ${ }^{25}$ using Monte-Carlo simulations.

To assess the performance of the tomography and radiography setup we estimate the spatial resolution and the contrast sensitivity using line-pair structures (line pairs per $\mathrm{mm}$ - LPM). ${ }^{26}$ In practice, we measured the contrast of a standard grid against the background provided by a homogenous object. Such a grid is usually made from stainless steel and contains both horizontal and vertical bars (see Fig. 3). The thickness and width of the bars are equal and are also equal to the spacing between consecutive bars, which allows us to estimate the spatial resolution as well as the contrast sensitivity. ${ }^{6,26}$ For our estimates, the grid parameter $a$ is varied from $2 \mathrm{~mm}$ to $0.1 \mathrm{~mm}(0.25 \mathrm{LPM}$ to $5 \mathrm{LPM})$.

The setup we propose is a first generation tomography setup ("pencil-beam") with a highly collimated beam. Toyokawa et al. ${ }^{27}$ demonstrated the working principle of a highenergy LCS gamma-ray imaging system for industrial objects using a "pencil-beam" setup. They imaged several objects made of either low $\mathrm{Z}$ (concrete) or high $\mathrm{Z}$ ( $\mathrm{Pb}, \mathrm{Fe}$, $\mathrm{Cu}$ ) materials, or a combination of the two. ${ }^{6,27,28}$ The best spatial resolution attained using $10 \mathrm{MeV}$ LCS photons was $650 \mu \mathrm{m}$. Similar results were obtained at the HI $\gamma \mathrm{S}$ facility, where, using CCD-based gamma camera detection, they demonstrated a lateral resolution of $0.5 \mathrm{~mm}$ and a contrast sensitivity better than $6 \% .{ }^{29}$ At ELI-NP, we expect to achieve a better spatial resolution and higher contrast sensitivity for similar measurement times, since the intensity of the ELI-NP gamma beam will be a few orders of magnitude higher.

Figure 3 shows a schematic drawing of the experimental setup that was used in the analytical calculations and in simulations. The photons emitted from the interaction point pass through the ELI-NP collimator, leading to a highly collimated beam within the specifications. ${ }^{5}$ The photon beam crosses the object (uniform object plus the regular grid) and is detected by a large size detector after passing through another collimator. The collimator is used to define the beam width, which is strongly related to the resolution of the setup, and to reduce the influence of scattered photons.

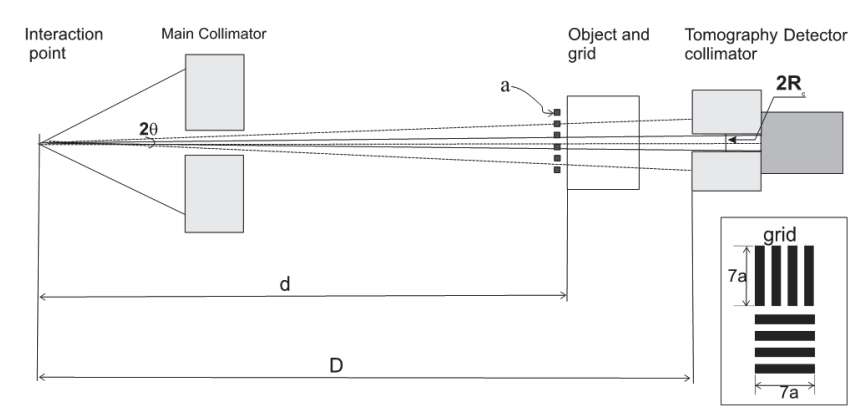

Fig. 3. Schematic drawing of the experimental setup and of the grid used to assess the resolution. 


\subsection{Analytical model}

In the following, we present the analytical model developed for the pencil-beam tomography setup used to estimate the resolution and counting rates in the detector as well as the optimal parameters to be used in the simulation/measurement. ${ }^{24}$

As we scan the beam across the grid (+object), the number of photons reaching the detector will vary between a minimum $\left(\mathrm{N}_{\mathrm{MIN}}\right)$ and a maximum $\left(\mathrm{N}_{\mathrm{MAX}}\right)$ as the beam intersects the grid bars and spaces. The number of photons reaching the detector is given by:

$$
\begin{gathered}
N_{M I N}=I_{0}\left(\frac{R_{C}}{D \theta}\right)^{2} e^{-\mu_{G} a} e^{-\mu x} t \epsilon, \\
N_{M A X}=I_{0}\left(\frac{R_{C}}{D \theta}\right)^{2} e^{-\mu x} t \epsilon,
\end{gathered}
$$

where $I_{0}$ is the source intensity, $\mathrm{R}_{\mathrm{C}}$ is the radius of the collimator placed after the object, $\mathrm{D}$ is the source-collimator distance, $\theta$ is the angular divergence of the beam, $t$ is the acquisition time, $\varepsilon$ is the efficiency of the detector, $\mu\left(\mu_{\mathrm{G}}\right)$ is the linear attenuation coefficient in the object (grid), and $x(a)$ is the object (grid) thickness.

In order to decide whether the grid is visible or not in the scan data, we need to compare the variation between $\mathrm{N}_{\mathrm{MAX}}$ and $\mathrm{N}_{\mathrm{MIN}}$. In practice, for very thin grids we only expect a few percent difference between $\mathrm{N}_{\mathrm{MAX}}$ and $\mathrm{N}_{\mathrm{MIN}}$, so a serious issue is whether the differences are significant from the point of view of counting statistics. To avoid this issue and obtain good counting statistics in the detector we introduce the following conditions:

$$
N_{M A X}-N_{M I N}>S \sqrt{N_{M A X}}
$$

where $\mathrm{S}$ is a significance factor. For $\mathrm{S}=2$ the error bars of the two extreme points will touch; therefore, a high contrast requires a larger value for S. Using Eqs. (1) through (3), we can express the measurement time as:

$$
t>\frac{s^{2}}{e^{-\mu x}}\left(\frac{d}{f a}\right)^{2} \frac{\theta^{2}}{I_{0} \epsilon} \frac{1}{\left(1-e^{-\mu_{G} a}\right)^{2}},
$$

where $f$ is a measure of how small the beam spot at the object is compared to the grid parameter $a$ and is defined by:

$$
\frac{R_{C}}{D}=\frac{f a}{d},
$$

Using Eq. (4), we can estimate the measurement time, maximum thickness, and desired count rates for any particular setup geometry and source intensity. Table 2 lists the thicknesses of several materials for which we can achieve $0.7 \mathrm{~mm}$ in resolution and in contrast sensitivity for exposures of one second at several energies. For instance, at the listed beam intensity and in one exposure, we need to wait only one second to have enough statistics to distinguish a $0.7 \mathrm{~mm} \mathrm{SS}$ wire (1.9\% contrast) in $40 \mathrm{~cm}$ of $\mathrm{Al}$ (for 3.5 $\mathrm{MeV}$ photons). The rates/macrobunch (number of photons within the bandwidth per 
$10 \mathrm{~ms}$ ) in the detector are listed in the table and are of the order of $10^{3}$ gammas. These values are estimated by using a single large detector $(\varepsilon=1)$. The maximum thickness of the investigated materials increases as we increase the energy of the gamma beam, mostly because of the improved parameters of the gamma beam at high energy. These numerical estimates are meant to give an idea about the expected performance of the radiography and tomography setup for a particular case. Higher resolutions and better contrast sensitivities than the ones mentioned can be achieved by varying the geometry, the object size, and the beam parameters. ${ }^{24}$

Table 2. Expected thickness of objects able to be scanned with a resolution of $0.7 \mathrm{~mm}^{*}(\mathrm{~d}=20 \mathrm{~m}, \mathrm{D}=40 \mathrm{~m})$. The first three rows list the main parameters of the gamma beam at several energies.

\begin{tabular}{lllll}
\hline Energy $(\mathrm{MeV})$ & 2 & 3.5 & 9.87 & 19.5 \\
\hline $\begin{array}{l}\text { Source divergence }(\mu \mathrm{rad}) \\
\text { Nr of photons within the FWHM }\end{array}$ & 140 & 100 & 50 & 40 \\
bandwidth & $4.0 \times 10^{8}$ & $3.7 \times 10^{8}$ & $8.3 \times 10^{8}$ & $8.1 \times 10^{8}$ \\
$\mathrm{Al}(\mathrm{cm})$ & 30 & 41.2 & 88.9 & 104.9 \\
$\mathrm{Fe}(\mathrm{cm})$ & 10.4 & 13.5 & 23.7 & 24.3 \\
$\mathrm{H}_{2} \mathrm{O}$ & 70.8 & 100.5 & 250.1 & 337.1 \\
Concrete $(\mathrm{cm})$ & 33.8 & 47 & 106.8 & 131.6 \\
Rates/macrobunch & $1.9 \times 10^{3}$ & $2.9 \times 10^{3}$ & $3.9 \times 10^{3}$ & $3.4 \times 10^{3}$ \\
\hline${ }^{*}$ Stainless steel $(\mathrm{SS})$ grid: $a=0.7 \mathrm{~mm} ; \mathrm{f}=0.5 ; \mathrm{R}_{\mathrm{C}}=0.7 \mathrm{~mm}$, i.e. the beam spot at the sample is as large as $a$.
\end{tabular}

\subsection{Numerical simulations}

To test the spatial resolution and the contrast sensitivity of the tomography setup, we have simulated the transmission through various objects and grids using Monte Carlo simulations implemented in GEANT4. ${ }^{22,23}$ The modeled gamma beam has a size between 10-30 $\mu \mathrm{m}$ and a divergence between 25-200 $\mu \mathrm{rad}$, depending on the energy of the beam (see Ref. 5). The simulations are validated by comparison with analytical calculations. ${ }^{24}$

Simulations were performed for the geometry outlined in Fig. 3 by using the regular grid as well as by using a complex phantom, as shown in Fig. 4. The phantom consists of a tube filled with aluminum that hosts a total of 12 rods of various materials and diameters ranging from $0.5 \mathrm{~mm}$ to $4 \mathrm{~mm}$. For the simulation of each exposure, we used a gamma beam of $3500 \pm 17 \mathrm{keV}$ and $100 \mu \mathrm{rad}$. The collimated beam is $0.5 \mathrm{~mm}$ in diameter at the object position (phantom), and we used $10^{6}$ photons $/ \mathrm{s}$ incident on the phantom.

Figure 4 shows the reconstructed image of the phantom. The spatial resolution and contrast sensitivity can be inferred from the reconstructed image and from line profiles. Sub-millimeter $(0.5 \mathrm{~mm})$ features are visible in all cases and are more pronounced for high-density materials (higher contrast). In order to discriminate between lower contrast parts (thin and low-density objects), a smaller beam spot is desirable, which implies a larger number of views and projections for a CT scan. A small beam spot $(<0.5 \mathrm{~mm})$ implies greatly reduced beam intensity for any conventional gamma source, but not for the future gamma beam at ELI-NP, which is inherently narrow (divergence between 
$200 \mu \mathrm{rad}$ and $40 \mu \mathrm{rad}$, see Table 2 and Ref. 5) and the perfect solution for high-resolution tomography.
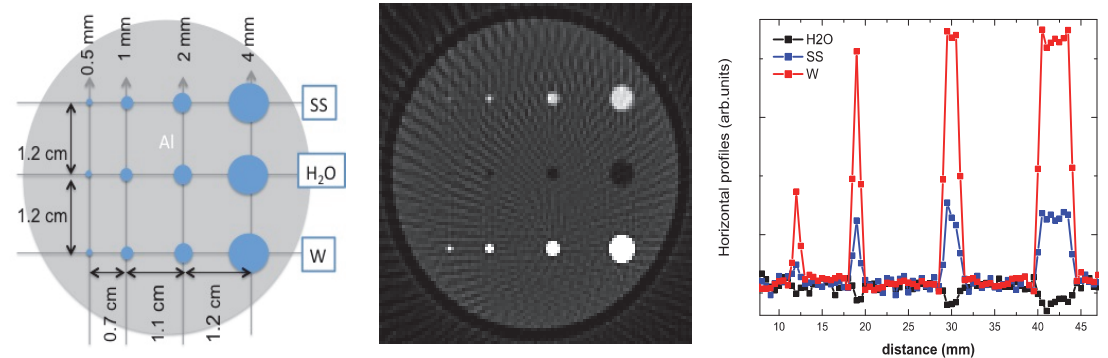

Fig. 4. CT reconstruction from simulated data using a filtered back-projection algorithm from 60 projections $\left(3^{\circ}\right)$ and 109 views $(0.5 \mathrm{~mm}$ step). A diagram of the phantom is shown on the left. Line profiles across the insets are also shown for better visualization of gamma attenuation (white corresponds to high attenuation). For this simulation, the source-object (-collimator) distance was $5 \mathrm{~m}(10 \mathrm{~m})$.

\section{Conclusion}

The gamma beam system at ELI-NP will deliver a very intense, quasi-monoenergetic, and energy-tunable gamma beam. By means of analytical calculations and numerical simulations, we demonstrate the possibility of using this gamma beam for industrial applications. The non-destructive assay based on NRF of nuclear materials can be successfully applied at ELI-NP, thanks to the high-intensity gamma source and the highefficiency detector array. The future gamma beam system will also be a perfect solution for industrial applications in radiography and tomography. The collimated beam intensity is a few orders of magnitude higher than any other gamma-ray source available worldwide, substantially increasing the maximum size of the investigated objects and the imaging resolution. Overall, the new gamma source at ELI-NP will deliver new opportunities not only for the research community but also for industry.

\section{Acknowledgments}

The ELI-NP project is co-funded by the European Union through the European Regional Development Fund. This work was supported by the Memorandum for Scientific Collaboration on the Implementation of the Extreme Light Infrastructure-Nuclear Physics (ELI-NP) Project between Horia Hulubei National Institute for R\&D in Physics and Nuclear Engineering (IFIN-HH), Romania and the Institute of Advanced Energy, Kyoto University, Japan.

\section{References}

1. C. A. Ur et al., Acta Physica Polonica B 46, 743 (2015).

2. ELI-Extreme Light Infrastructure Science and Technology with ultra-intense Lasers Whitebook, eds. G.A Mourou, G. Korn, W. Sandner, and J.L. Collier (THOSS Media GmbH 2011). 
3. ELI-NP White Book, http://www.eli-np.ro/documents/ELI-NP-WhiteBook.pdf.

4. D. Ursescu et al., High-power, high-energy, and high-intensity laser technology; and Research using extreme light: entering new frontiers with petawatt-class lasers, in Proc. of SPIE 8780, eds. G. Horn, J. Hein and L. O. Silva (2013) p. 87801H.

5. O. Adriani et al., Technical Design Report EuroGammaS proposal for the ELI-NP Gamma beam System, arXiv:1407.3669.

6. H. Toyokawa et al., Nucl. Instr. Meth. Phys. Res. A 608, S41 (2009).

7. H. Toyokawa et al., Japanese Journal of Applied Physics 50, 100209 (2011).

8. H. R. Weller et al., Prog. Part. Nucl. Phys. 62, 257 (2009).

9. W. Bertozzi and R. J. Ledoux, Nucl. Instr. Meth. Phys. Res. B 241, 820 (2005).

10. J. Pruet et al., J. Appl. Phys. 99, 123102 (2006).

11. C. A. Hagmann et al., J. of Appl. Phys. 106, 084901 (2009).

12. B. J. Quiter, Bernhard A. Ludewigt, Vladimir V. Mozin, and Stanley G. Prussin, IEEE Trans. on Nucl. Sci. 58, 400 (2011).

13. G. Warren, J. Caggiano, and P. Peplowski, AIP Conference Proceedings 1194, 106 (2009).

14. O. Beck et. al., J. Appl. Phys. 83, 5484 (1998).

15. M. N. Lakshmanan, B. P. Harrawood, G. A. Agasthya, and A. J. Kapadia, IEEE Trans. Med. Imag. 33, 546 (2014).

16. H. Yang, Active interrogation methods for detection of special nuclear material, Thesis, url: http://hdl.handle.net/2027.42/64778.

17. T. Hayakawa et al., Rev. Sci. Instr. 80, 045110 (2009).

18. B.A. Ludewigt, B. J. Quiter, and S.D. Ambers, Nuclear Resonance Fluorescence for Safeguards Applications, DOE report, http://dx.doi.org/10.2172/1022713 (2011).

19. Nuclear Resonance Fluorescence at ELI-NP, Technical Design Report, to be published.

20. M. A. Schumaker and C.E. Svensson, Nucl. Instr. Meth. Phys. Res. A 575, 421 (2007).

21. M. A. Schumaker et al., Nucl. Instr. Meth. Phys. Res. A 570, 437 (2007).

22. S. Agostinelli et al., Nucl. Instr. Meth. Phys. Res. A 506, 250 (2003).

23. J. Allison et al., IEEE Trans. Nucl. Sci. 53, 270 (2006).

24. Gamma Beam Industrial Applications at ELI-NP, Technical Design Report, to be published.

25. I. Daito et al., Simulation Study on Computer Tomography Imaging of Nuclear Distribution by Quasi Monoenergetic Gamma Rays with Nuclear Resonance Fluorescence: case study for ELINP application, to be published in Proc. 2015 Eco-Energy and Materials Science and Eng. Symp. (2015).

26. A. Staude and J. Goebbels, Determining the Spatial Resolution in Computed Tomography Comparison of MTF and Line-Pair Structures, in International Symposium on Digital Industrial Radiology and Computed Tomography - Tu.4.1.

27. H. Toyokawa, H. Ohgaki, T. Mikado, and K. Yamada, Rev. Sci. Instr. 73, 3358 (2002).

28. H. Toyokawa et al., Proc. Part. Acc. Conf. 713 (2003).

29. C. Sun, Characterizations and Diagnostics of Compton Light Source, PhD Thesis, Duke University (2009). 